\title{
ACTION LEVELS FOR SARS-COV-2 IN AIR: PRELIMINARY APPROACH
}

\author{
Charles N. Haas \\ Department of Civil, Architectural and Environmental Engineering \\ Drexel University \\ Philadelphia, PA 19104 \\ haas@drexel.edu
}

August 14, 2020

\begin{abstract}
Quantitative microbial risk assessment has been used to develop criteria for exposure to many microorganisms via various exposures. In this paper the dose response curve for Coronavirus $229 \mathrm{E}$ is used to develop preliminary risk based exposure criteria for SARS-CoV-2 via the respiratory portals of entry.
\end{abstract}

Keywords SARS-CoV-2 - risk assessment · QMRA

\section{Introduction}

SARS-CoV-2 has emerged as a significant pathogen of global concern. There has been increasing recognition of the role of the respiratory system as a portal of entry. There has been a historical differentiation between transmission by "droplets" and transmission by smaller particles[1]. However more recent understanding is differentiating between direct deposition of particles emitted via an infected person onto moist surfaces (nose, mouth, etc.) of a susceptible person (sometimes also called short range transmission) versus particles that may circulate in (particularly indoor) air before being inhaled by a susceptible person[2]. This is more consistent with understanding of how particles behave in the air[3, 4].

The importance of both shorter range and longer range routes of exposure for community transmission has been borne out in studies of outbreaks, in particular the Diamond Princess cruise ship [5] and the Skagit WA choir cluster[6]. Modeling studies also indicate that both routes are important in the health care setting[7].

For both of the above mechanisms, the portal of entry is the respiratory system. However the size of the particle will influence its deposition within the respiratory tract[8].

There is also a potential portal of entry via short range deposition to the eye [9, 10]. However this portal of entry will not be considered in this paper.

A critical task for determination of control strategies is to determine doses that would be regarded as acceptable. Then the corresponding environmental (e.g. air) concentrations can be computed. By comparing these to the air concentrations resulting from uncontrolled settings in the presence of sources (one of more infected individuals emitting virus), one can then determine the magnitude of source reduction needed. This can be implemented in a variety of ways, including a layered, or multiple barrier, approach consisting of use of masks, ventilation, and active air treatment systems.

The objective of this paper is to provide a preliminary framework to the determination of acceptable air levels of virus using a quantitative microbial risk assessment (QMRA) approach[11]. 


\section{Approach}

Watanabe et al.[12] reviewed the literature for available data sets (human and animal) for development of dose-response models for various coronaviruses. Of these, there was only one human data set (with Coronavirus 229E) and this had the lowest median effective dose (most potent). This will be used in this analysis.

The underlying data were from experimental work by Bradburne et al.[13] in which human volunteers were dosed into their nostrils with different amounts of virus. The endpoint response was illness. Watanabe et al. [12] found that the exponential dose response model (Equation 1) provided good fit to the data.

$$
p=1-\exp \left(-\frac{d}{k}\right)
$$

In this equation, $\mathrm{d}$ is the average dose (in this case, plaque forming units - pfu - of virus), $\mathrm{k}$ is the dose-response parameter (interpreted as the inverse of the probability that one virus will survive and initiate the endpoint effect), and $p$ is the risk.

There are multiple, equivalent, ways of interpreting p. It can be regarded as the probability than a single individual exposed to the average dose of $\mathrm{d}$ will have the effect. It can also be interpreted as the expected proportion of individuals exposed to the average dose of $\mathrm{d}$ who will have the adverse effect. Finally (and in the interpretation used below), it can be regarded as the number of individuals who would need to be exposed to the average dose such that one adverse case would be expected.

The fit to the dose response data will be used to ascertain the number of individuals exposed to a particular average dose that would be required to observe at least one adverse case.

\section{Results}

The Watanabe et al.[12] fit to the data of Bradburne et al.[13] is shown in Figure 1] The best fit value of $\mathrm{k}$ is 18.54, indicating that each pfu has a probability of 0.054 of causing an adverse effect.

By taking the reciprocal of "p" in Equation 1, we can calculate the number of persons who would be required to be exposed to an average dose in order to expect to observe one case. This analysis is shown in Figure 2 Note that this is a straight line with a slope of unity on a log-log plot since at low doses, the exponential dose-response relationship is closely approximated by a linear relationship.

As an example of the use of Figure 2 if 100 people are of interest, then an average exposed dose of less than 0.18 pfu would be needed to be below the critical line. This could be translated into an air concentration if a breathing rate and duration was specified.

As an elaboration, if the time of exposure was one hour, and if a light intensity breathing rate of $0.012 \frac{\mathrm{m}^{3}}{\mathrm{~min}}$ was used (https://www.epa.gov/sites/production/files/2015-09/documents/efh-chapter06.pdf), the dose corresponds to a virus concentration of $0.25 \frac{p f u}{m^{3}}$.

An alternative or complementary approach to viewing the data is to look at the probability of having one or more cases given an average dose and population size. This is shown in Figure 3. So for example, if 100 people are exposed and it is desired to keep the risk of a case below 1\%, then the maximum average dose must be below $0.0011 \mathrm{pfu}$.

\section{Discussion and Limitations}

This analysis uses 229E as the coronavirus for reference. This is an alphacoronavirus, in contrast to SARS-CoV-2, which is a betacoronavirus [14]. Whether the betacoronaviruses have substantially different dose-response behavior remain to be seen, and would likely require testing in suitable animal models. The other viruses analyzed by Watanabe et al.[12] show lesser potency than 229E.

This report develops relationships based on viable infectious virus (pfu). Most environmental studies have performed measurements based on gene copies $(\mathrm{gc})$ of viral RNA isolated. The relationship between the two, and the effect of holding conditions on this relationship is not known.

A study in a hospital in Nebraska observed up to $50 \mathrm{gc} / \mathrm{L}$ in air samples in COVID19 patient areas[15]. Some of the environmental samples showed cypopathogenic effects (CPE) in tissue culture, although confirmation of SARS-CoV-2 could not be attempted. 


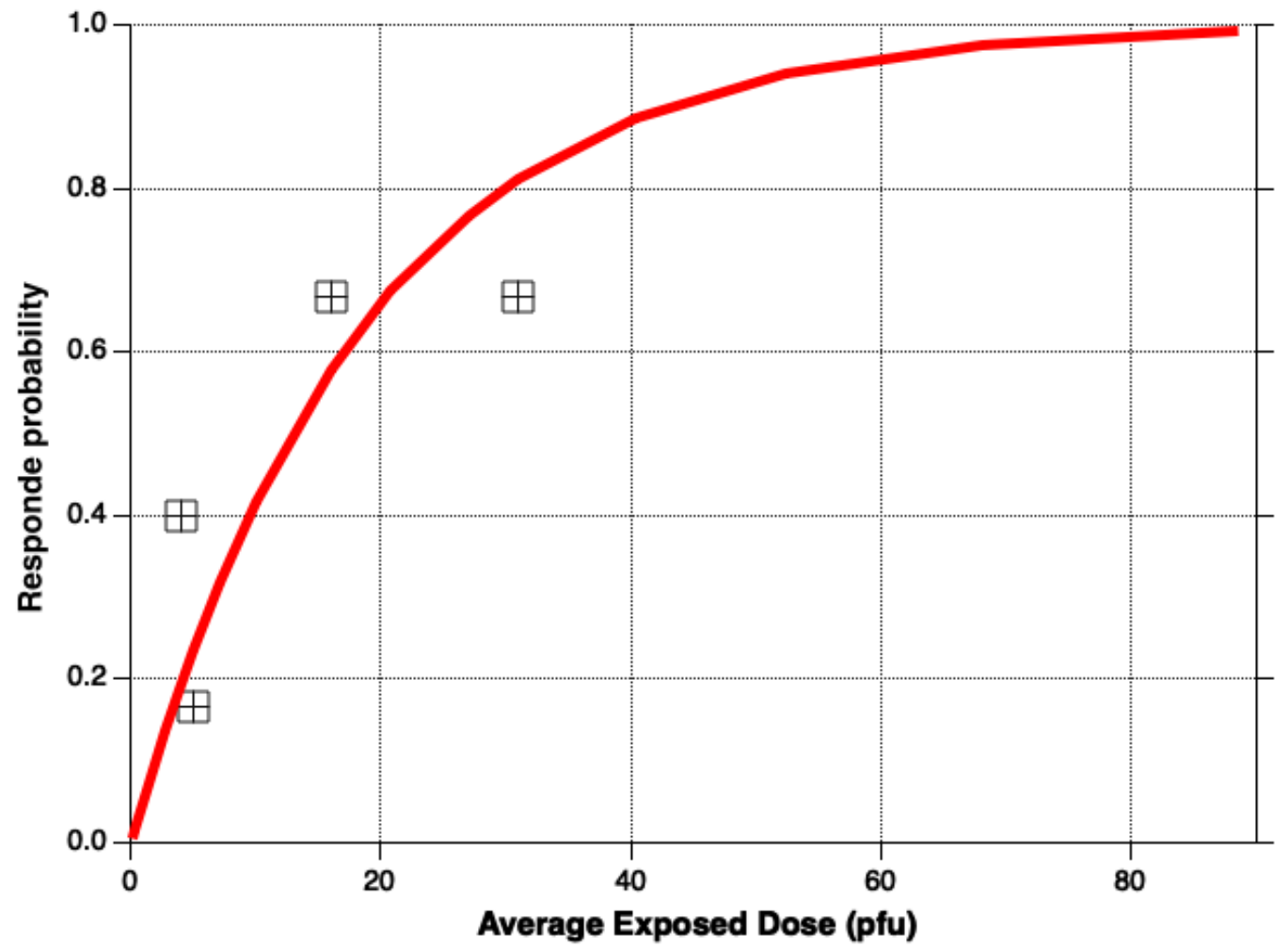

Figure 1: Fit of exponential dose response model (curve) to human trial of coronavirus 229E. Data (points) from[13]

Lednicky et al. [16] found $0.9 \mathrm{gc} / \mathrm{L}$ of SARS-CoV-2 in air samples in a student clinic. When cultured, CPE was observed, although confirmation of the virus in the tissue culture was not performed.

In hospitalized patients it was observed that swab samples were not positive for viable virus until PCR titers were in excess of $10^{6}$ RNA copies [17]. It is known, however, that virus can be produced in substantial numbers in presymptomatic cases, perhaps at even greater amounts than in symptomatic and hospitalized cases[18]. Therefore a "typical" value for a ratio between viable infectious virus and gene copies in environmental samples remains unclear.

The underlying dose-response study used in this development was based on direct placement of drops in the nostrils. The impact and potential differences associated with inhalation of smaller particles which may have a greater chance of deeper penetration into the respiratory tract remains uncertain.

\section{Conclusions}

There is enough information to develop preliminary guideposts for the dose of SARS-CoV-2 that would present an acceptable risk. Based on standard assumptions of breathing rate and duration of exposure, this can be translated into air concentration. With this, estimation of source strength, and standard mass balance models of indoor air quality [19], the specification of needed interventions to control risk from air exposure can be undertaking.

Clearly there are data gaps, and as new information emerges the refinement of this approach can occur. However in the design of interventions, it is important not to let the perfect be the enemy of the good, and it becoming clear that we have quantitative tools to assist in the design of engineering interventions.

\section{References}

[1] Kevin P Fennelly. Particle sizes of infectious aerosols: Implications for infection control. The Lancet Respiratory Medicine, Huly 242020. 


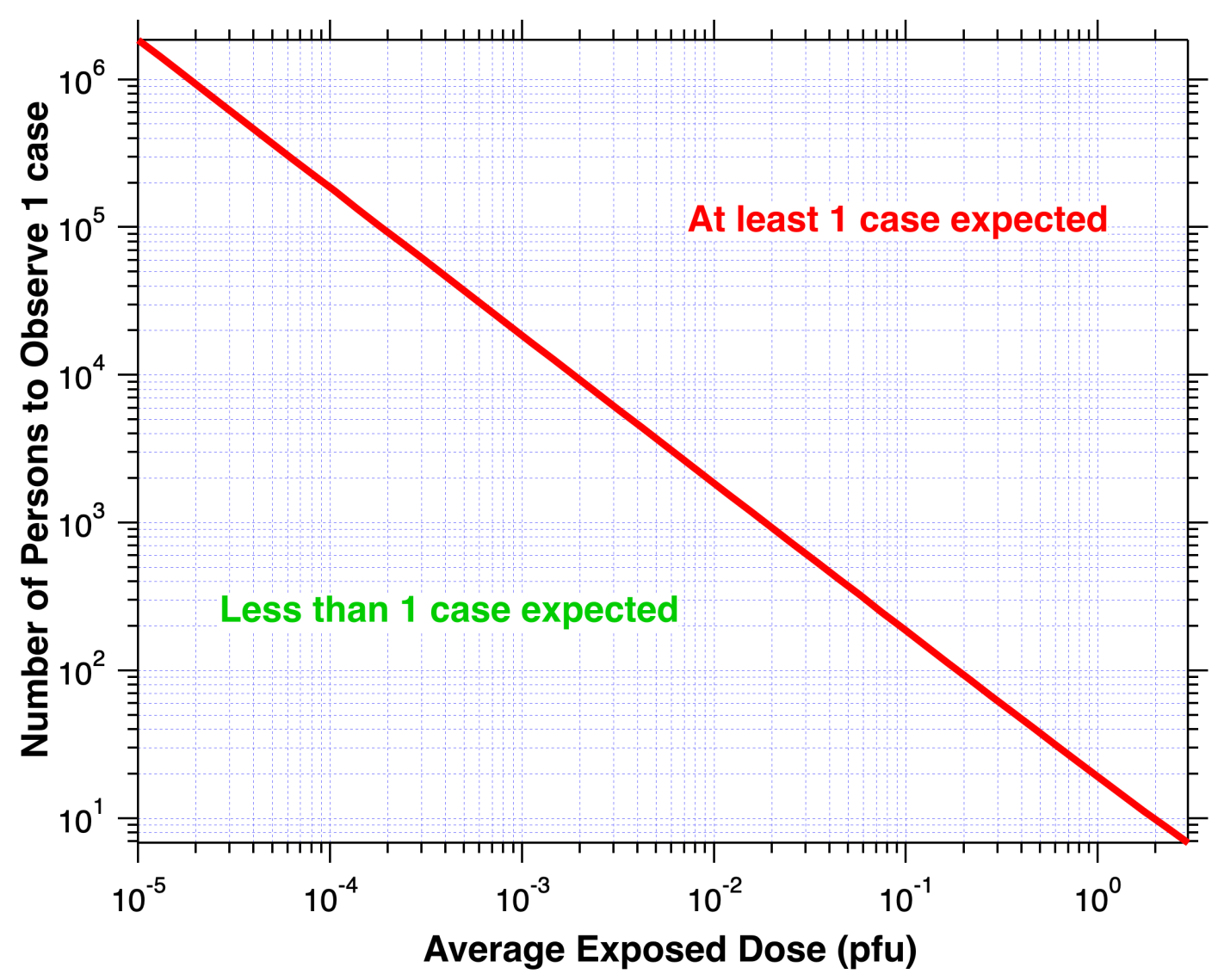

Figure 2: Critical Line for One Observed Case

[2] Donald K Milton. A Rosetta Stone for Understanding Infectious Drops and Aerosols. Journal of the Pediatric Infectious Diseases Society, page piaa079, July 2020.

[3] Lidia Morawska, Julian W. Tang, William Bahnfleth, Philomena M. Bluyssen, Atze Boerstra, Giorgio Buonanno, Junji Cao, Stephanie Dancer, Andres Floto, Francesco Franchimon, Charles Haworth, Jaap Hogeling, Christina Isaxon, Jose L. Jimenez, Jarek Kurnitski, Yuguo Li, Marcel Loomans, Guy Marks, Linsey C. Marr, Livio Mazzarella, Arsen Krikor Melikov, Shelly Miller, Donald K. Milton, William Nazaroff, Peter V. Nielsen, Catherine Noakes, Jordan Peccia, Xavier Querol, Chandra Sekhar, Olli Seppänen, Shin-ichi Tanabe, Raymond Tellier, Kwok Wai Tham, Pawel Wargocki, Aneta Wierzbicka, and Maosheng Yao. How can airborne transmission of COVID-19 indoors be minimised? Environment International, 142:105832, September 2020.

[4] Kimberly A. Prather, Chia C. Wang, and Robert T. Schooley. Reducing transmission of SARS-CoV-2. Science, 2020.

[5] Parham Azimi, Zahra Keshavarz, Jose Guillermo Cedeno Laurent, Brent R. Stephens, and Joseph G. Allen. Mechanistic Transmission Modeling of COVID-19 on the Diamond Princess Cruise Ship Demonstrates the Importance of Aerosol Transmission. Preprint, Infectious Diseases (except HIV/AIDS), July 2020.

[6] Shelly L Miller, William W Nazaroff, Jose L Jimenez, Atze Boerstra, Giorgio Buonanno, Stephanie J Dancer, Jarek Kurnitski, Linsey C Marr, Lidia Morawska, and Catherine Noakes. Transmission of SARS-CoV-2 by inhalation of respiratory aerosol in the Skagit Valley Chorale superspreading event. Preprint, Infectious Diseases (except HIV/AIDS), June 2020.

[7] Rachael M. Jones. Relative contributions of transmission routes for COVID-19 among healthcare personnel providing patient care. Journal of Occupational and Environmental Hygiene, pages 1-8, July 2020.

[8] A. E. Haddrell, J. F. Davies, and J. P. Reid. Dynamics of Particle Size on Inhalation of Environmental Aerosol and Impact on Deposition Fraction. Environ Sci Technol, November 2015. 


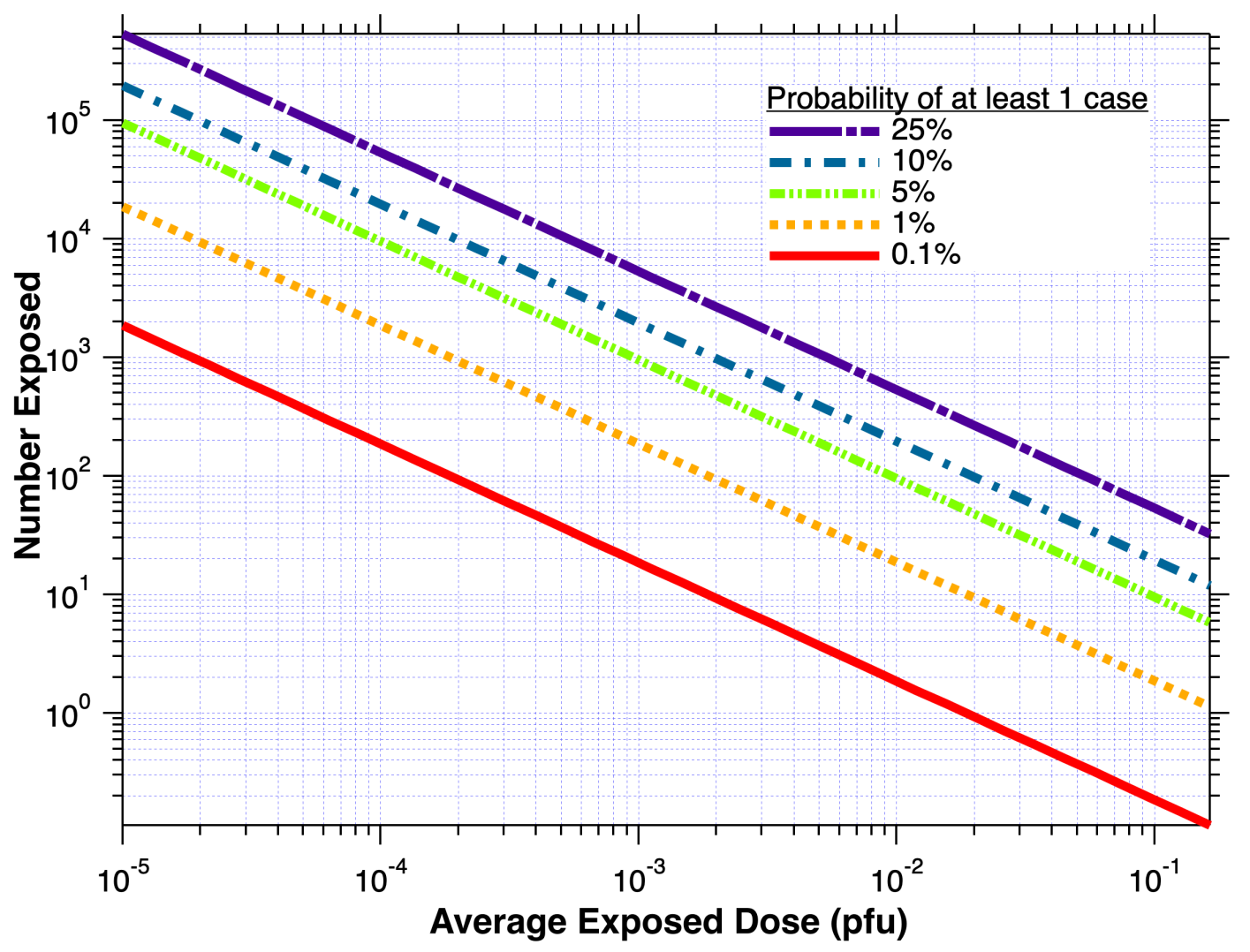

Figure 3: Probability of 1 or More Cases as a Function of Average Exposure and Number Exposed

[9] Derek K Chu, Elie A Akl, Stephanie Duda, Karla Solo, Sally Yaacoub, and Holger J Schünemann. Physical distancing, face masks, and eye protection to prevent person-to-person transmission of SARS-CoV-2 and COVID19: A systematic review and meta-analysis. The Lancet, 395(10242):1973-1987, 2020.

[10] C Raina MacIntyre and Quanyi Wang. Physical distancing, face masks, and eye protection for prevention of COVID-19. The Lancet, 395(10242):1950-1951, June 2020.

[11] Charles N. Haas, Joan B. Rose, and Charles P. Gerba. Quantitative Microbial Risk Assessment. John Wiley, New York, second edition, 2014.

[12] Toru Watanabe, Timothy A. Bartrand, Mark H. Weir, Tatsuo Omura, and Charles N. Haas. Development of a Dose-Response Model for SARS Coronavirus. Risk Analysis, 30:1129-1138, 2010.

[13] A. F. Bradburne, M. L. Bynoe, and D. A. Tyrrell. Effects of a "new" human respiratory virus in volunteers. BMJ, 3(5568):767-769, September 1967.

[14] Ding X. Liu, Jia Q. Liang, and To S. Fung. Human Coronavirus-229E, -OC43, -NL63, and -HKU1. In Reference Module in Life Sciences, page B978012809633821501X. Elsevier, 2020.

[15] Joshua L. Santarpia, Danielle N. Rivera, Vicki L. Herrera, M. Jane Morwitzer, Hannah M. Creager, George W. Santarpia, Kevin K. Crown, David M. Brett-Major, Elizabeth R. Schnaubelt, M. Jana Broadhurst, James V. Lawler, St. Patrick Reid, and John J. Lowe. Aerosol and surface contamination of SARS-CoV-2 observed in quarantine and isolation care. Scientific Reports, 10(1):12732, December 2020.

[16] John A. Lednicky, Sripriya N. Shankar, Maha A. Elbadry, Julia C. Gibson, Md. Mahbubul Alam, Caroline J. Stephenson, Arantzazu Eiguren-Fernandez, J. Glenn Morris, Carla N. Mavian, Marco Salemi, James R. Clugston, and Chang-Yu Wu. Collection of SARS-CoV-2 Virus from the Air of a Clinic within a University Student Health Care Center and Analyses of the Viral Genomic Sequence. Aerosol and Air Quality Research, 20(6):1167-1171, 2020.

[17] Jeroen J.A. van Kampen, David A.M.C. van de Vijver, Pieter L.A. Fraaij, Bart L. Haagmans, Mart M. Lamers, Nisreen Okba, Johannes P.C. van den Akker, Henrik Endeman, Diederik A.M.P.J. Gommers, Jan J. Cornelissen, 
Rogier A.S. Hoek, Menno M. van der Eerden, Dennis A. Hesselink, Herold J. Metselaar, Annelies Verbon, Jurriaan E.M. de Steenwinkel, Georgina I. Aron, Eric C.M. van Gorp, Sander van Boheemen, Jolanda C. Voermans, Charles A.B. Boucher, Richard Molenkamp, Marion P.G. Koopmans, Corine Geurtsvankessel, and Annemiek A. van der Eijk. Shedding of infectious virus in hospitalized patients with coronavirus disease-2019 (COVID-19): Duration and key determinants. Preprint, Infectious Diseases (except HIV/AIDS), June 2020.

[18] Melissa M. Arons, Kelly M. Hatfield, Sujan C. Reddy, Anne Kimball, Allison James, Jesica R. Jacobs, Joanne Taylor, Kevin Spicer, Ana C. Bardossy, Lisa P. Oakley, Sukarma Tanwar, Jonathan W. Dyal, Josh Harney, Zeshan Chisty, Jeneita M. Bell, Mark Methner, Prabasaj Paul, Christina M. Carlson, Heather P. McLaughlin, Natalie Thornburg, Suxiang Tong, Azaibi Tamin, Ying Tao, Anna Uehara, Jennifer Harcourt, Shauna Clark, Claire Brostrom-Smith, Libby C. Page, Meagan Kay, James Lewis, Patty Montgomery, Nimalie D. Stone, Thomas A. Clark, Margaret A. Honein, Jeffrey S. Duchin, and John A. Jernigan. Presymptomatic SARS-CoV-2 Infections and Transmission in a Skilled Nursing Facility. New England Journal of Medicine, 382(22):2081-2090, May 2020.

[19] W. W. Nazaroff. Indoor bioaerosol dynamics. Indoor Air, 26(1):61-78, 2014. 